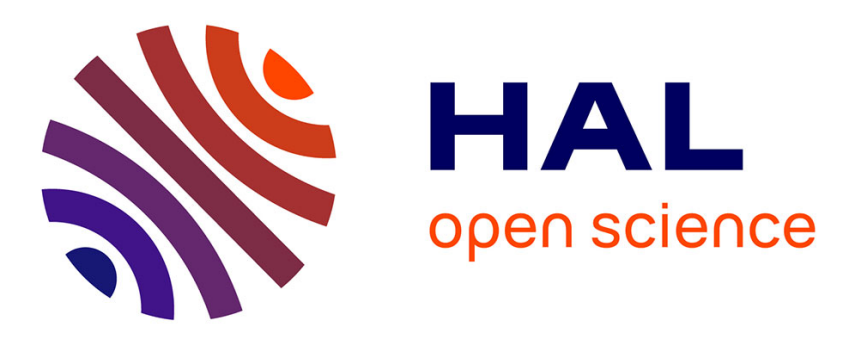

\title{
Mise en évidence des sous-structures de fluage par microanalyseur à sonde électronique
}

\author{
F. Maurice, P. Dorizzi, J.-P. Poirier
}

\section{To cite this version:}

F. Maurice, P. Dorizzi, J.-P. Poirier. Mise en évidence des sous-structures de fluage par microanalyseur à sonde électronique. Revue de Physique Appliquée, 1974, 9 (2), pp.399-401. 10.1051/rphysap:0197400902039900 . jpa-00243794

\section{HAL Id: jpa-00243794 https://hal.science/jpa-00243794}

Submitted on 1 Jan 1974

HAL is a multi-disciplinary open access archive for the deposit and dissemination of scientific research documents, whether they are published or not. The documents may come from teaching and research institutions in France or abroad, or from public or private research centers.
L'archive ouverte pluridisciplinaire HAL, est destinée au dépôt et à la diffusion de documents scientifiques de niveau recherche, publiés ou non, émanant des établissements d'enseignement et de recherche français ou étrangers, des laboratoires publics ou privés. 


\title{
MISE EN ÉVIDENCE DES SOUS-STRUCTURES DE FLUAGE PAR MICROANALYSEUR A SONDE ÉLECTRONIQUE
}

\author{
Mile F. MAURICE, Agent CEA \\ M. P. DORIZZI, Stagiaire SRMP \\ M. J.-P. POIRIER, Agent CEA
}

\begin{abstract}
Résumé. - On décrit une méthode de mise en évidence de la sous-structure de fluage au moyen d'images électroniques réalisées sur un microanalyseur à sonde électronique.

L'examen de monocristaux Au, Ag, Alliage Au-Ag permet une étude qualitative de la sousstructure et même une approche semi-quantitative de la désorientation des sous-grains.
\end{abstract}

\begin{abstract}
We describe a method of investigation of the creep sub-structure by means of electronic images realized with an electron microprobe. A qualitative study of the sub-structure and a semi quantitative study of the sub-grains misorientation is made on single crystals of $\mathrm{Au}, \mathrm{Ag}$ and Au-Ag alloys.
\end{abstract}

1. Introduction. - Le fluage à haute température fait apparaître une vitesse de déformation quasi stationnaire résultant d'un compromis entre la consolidation du matériau et sa restauration par montée des dislocations. Le fluage stationnaire est caractérisé par une sous-structure de polygonisation formée de cellules faiblement désorientées séparées par des parois de dislocations.

Les méthodes d'observation des sous-structures de fluage sont traditionnellement la méthode de Berg-Barrett qui ne donne de l'éprouvette qu'une image au grandissement 1 et les figures d'attaque ne révélant que les sous-joints.

La méthode que nous préconisons c'est-à-dire la visualisation de la sous-structure au moyen d'images électroniques réalisées sur un microanalyseur à sonde électronique permet d'observer l'éprouvette à des grandissements variables. Les images ainsi obtenues possèdent une grande sensibilité à la désorientation.

L'examen de ces images permet non seulement d'obtenir des renseignements qualitatifs mais aussì de tenter une approche semi-quantitative par la mesure de la désorientation relative des blocs.

2. Conditions expérimentales. - Nous avons examiné des monocristaux d'Au, $\mathrm{Ag}$, alliage $\mathrm{Au}-\mathrm{Ag}$ de forme parallélépipédique ayant pour faces des plans du type (001), (110) (110).

Les échantillons sont flués par compression suivant l'axe [001] à des températures supérieures à $0,5 T_{\mathrm{f}}$.

La déformation a lieu par glissement sur les plans du type (101) et (101) dans les directions [101] et [101].

Les figures 1,2 et 3 montrent les images obtenues sur $\mathrm{Au}, \mathrm{Ag}, \mathrm{Ag}-\mathrm{Au}$ en enregistrant les variations

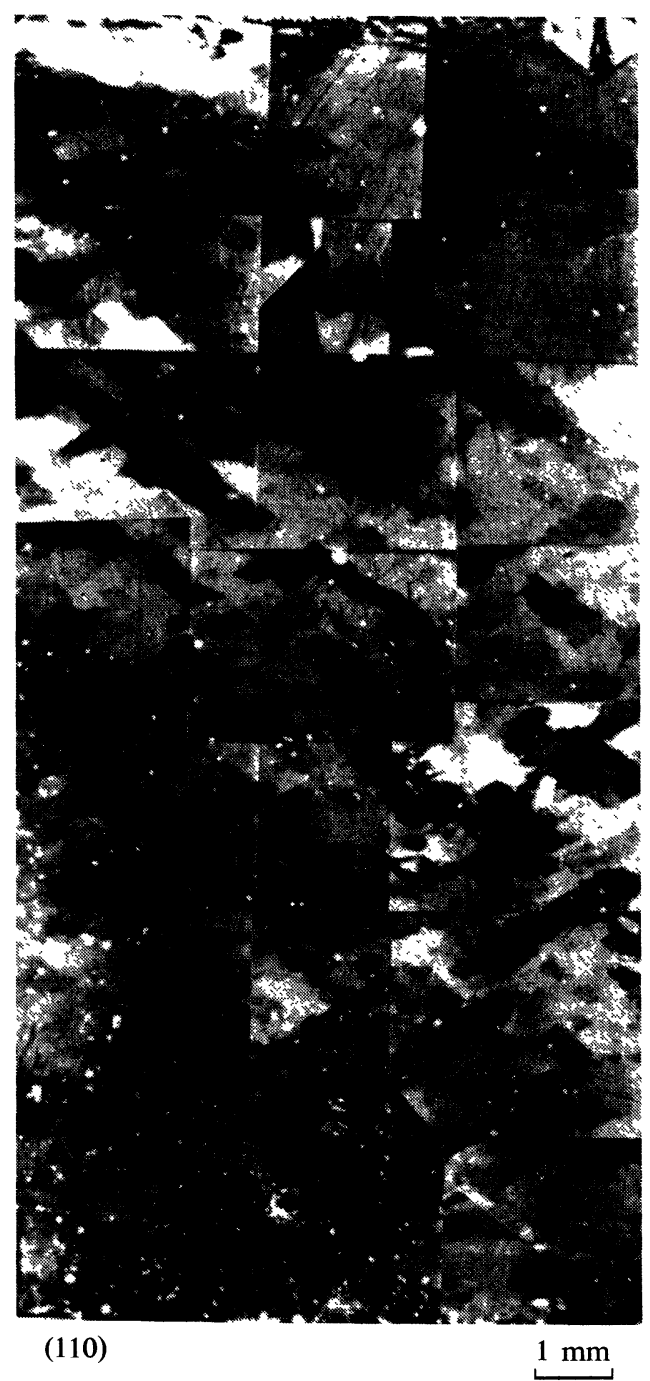

Fig. 1. - Image de la sous-structure d'un monocristal d'Au flué à $816^{\circ} \mathrm{C}$ sous une contrainte de $160 \mathrm{~g} / \mathrm{mm}^{2} . \varepsilon=4,5 \%$. 


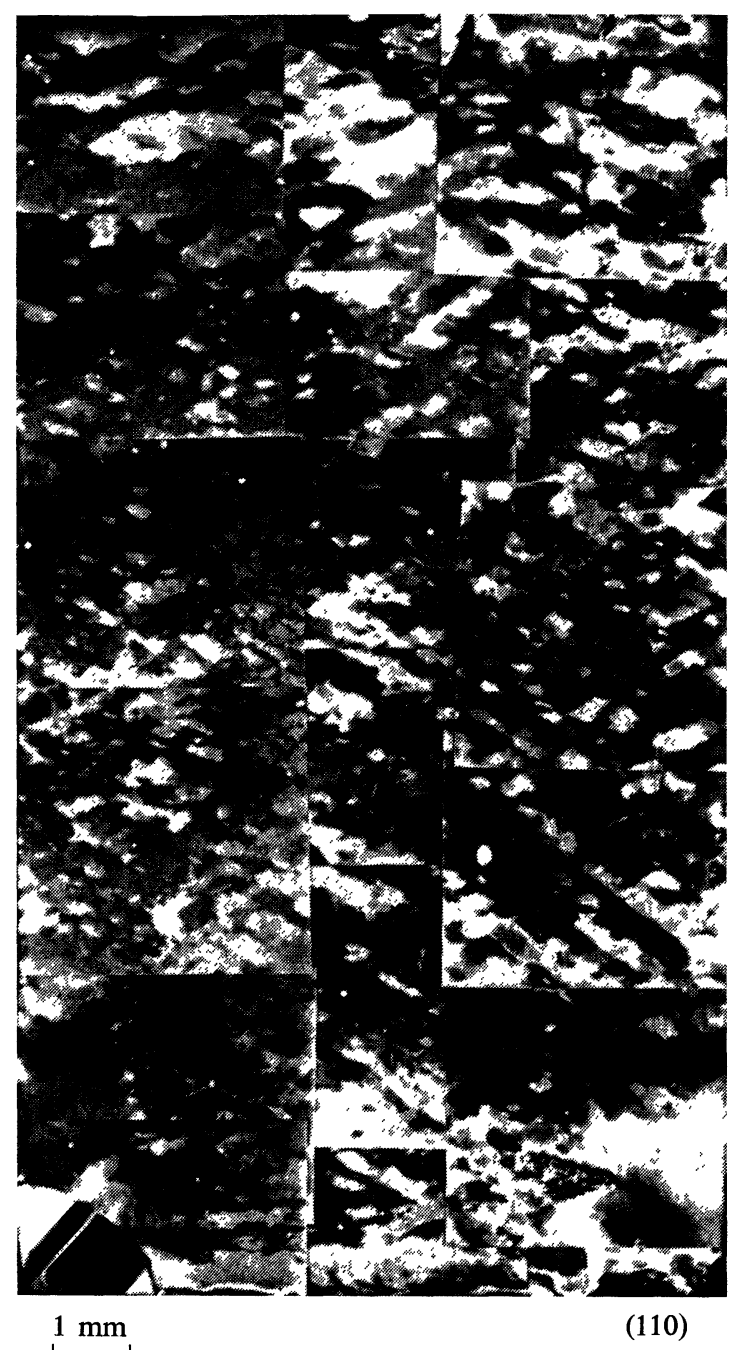

FIG. 2. - Image de la sous-structure d'un monocristal d'Ag flué à $880{ }^{\circ} \mathrm{C}$ sous une contrainte de $175 \mathrm{~g} / \mathrm{mm}^{2} . \varepsilon=3,75 \%$.

du courant absorbé par l'échantillon lorsque le faisceau d'électrons balaye la face latérale (110) dans les deux directions orthogonales [001] et [110].

L'échantillon est situé à $70 \mathrm{~mm}$ en dessous du plan normal de focalisation de façon à diminuer le grandissement (on réduit ainsi d'un facteur 10 le grandissement : $25 \times$ au lieu de $250 \times$ ). La plage balayée est alors de $2,5 \mathrm{~mm} \times 2,5 \mathrm{~mm}$, l'angle de balayage est de $\pm 1,55 \times 10^{-2}$ rd et la divergence du faisceau inférieure à $4 \times 10^{-3} \mathrm{rd}$. Les conditions de fonctionnement du microanalyseur sont $20 \mathrm{kV}$, $40 \mathrm{nA}$.

Le contraste des images est dû aux effets d'absorption ou de transmission anormale au voisinage de la réflexion de Bragg [3]. Il est de même nature que celui donnant naissance aux diagrammes de pseudo Kikuchi.

Dans notre cas particulier les deux réflexions de bas indice, que l'on peut obtenir sur la face (110) sont les réflexions 002 et $\overline{2} 20$ correspondant à des angles de Bragg $\Theta_{\mathrm{BO02}}=2,1 \times 10^{-2} \mathrm{rd}$ et $\Theta_{\mathbf{B} \overline{2} 20}=3 \times 10^{-2} \mathrm{rd}$.

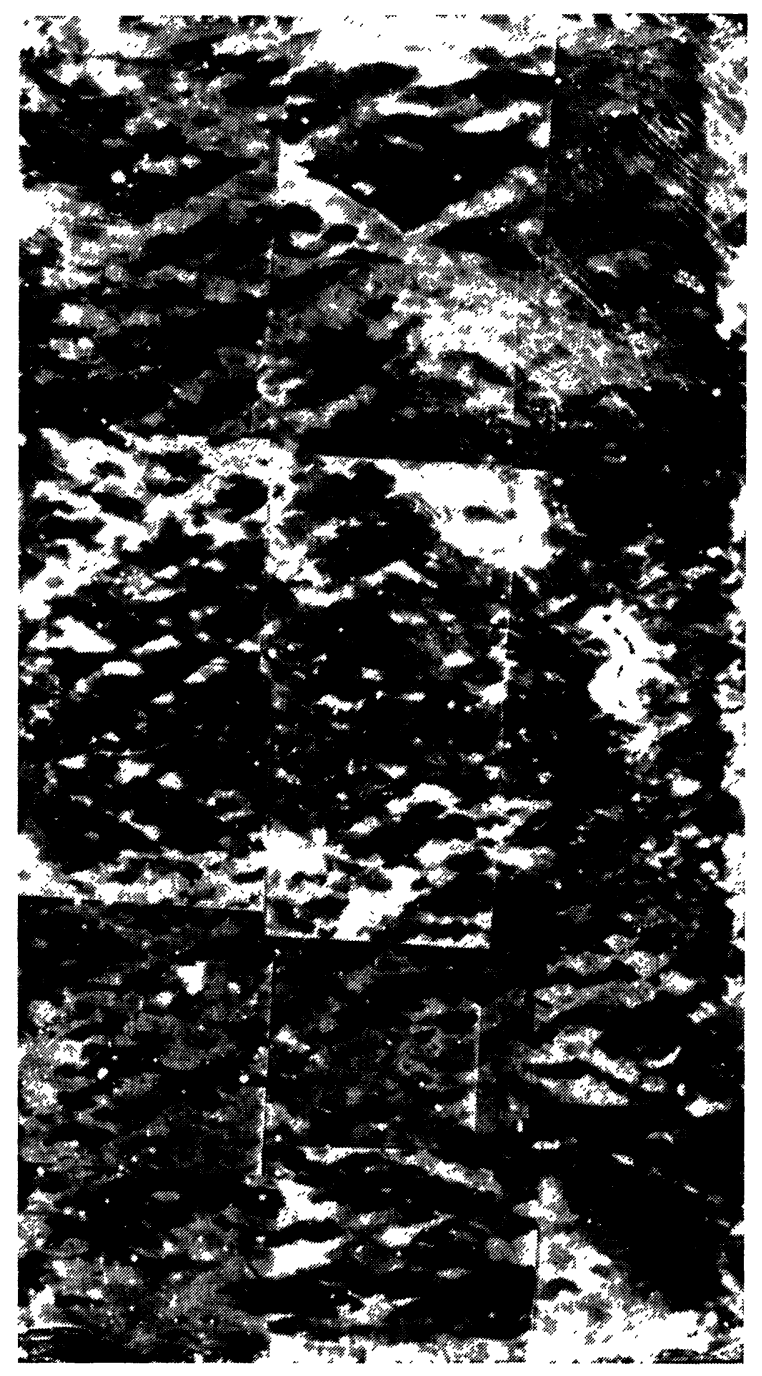

$1 \mathrm{~mm}$

(110)

FIG. 3. - Image de la sous-structure d'un monocristal d'alliage Au-Ag flué à $874{ }^{\circ} \mathrm{C}$ sous une contrainte de $224 \mathrm{~g} / \mathrm{mm}^{2} . \varepsilon=8,5 \%$.

Puisque l'angle de balayage $\left(\alpha=1,55 \times 10^{-2} \mathrm{rd}\right)$ est inférieur aux angles de Bragg, le contraste que nous observons est dû à la désorientation des sousgrains à l'intérieur d'une même bande.

3. Examen de la sous-structure. - 3.1 ETUDE QUALITATIVE. - L'observation de la sous-structure de fluage de monocristaux $\mathrm{Au}, \mathrm{Ag}$, alliage $\mathrm{Au}-\mathrm{Ag}$ permet de dégager les faits suivants [1]:

- La sous-structure est composée de cellules localisées dans les zones où le glissement $\{110\}$ peut opérer pour les faibles déformations et uniformément réparties dans toute l'éprouvette pour les fortes déformations (Fig. 4).

- Les sous-grains sont disposés en bandes parallèles à la trace des plans de glissement $\{110\}$ dans les régions où ces plans peuvent glisser librement.

- L'examen de la sous-structure sur les faces (110) et $(001)$ permet de conclure que les cellules sont sépa- 


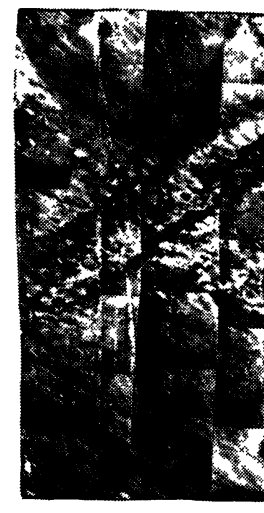

(110)

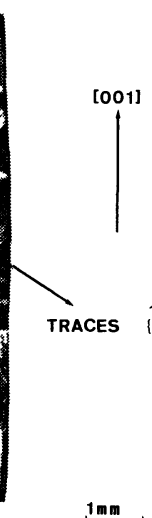

$1 \mathrm{~mm}$

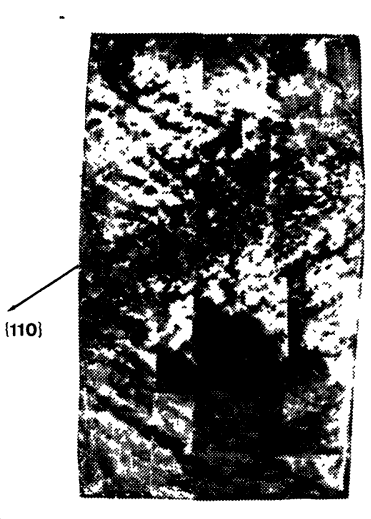

(110)

Fig. 4. - Evolution de la sous-structure suivant la contrainte appliquée. a) $T=816^{\circ} \mathrm{C} ; \varepsilon=3 \%, \sigma=140 \mathrm{gf} / \mathrm{mm}^{2} ; b$ ) $T=816^{\circ} \mathrm{C}, \varepsilon=10 \%, \sigma=225 \mathrm{gf} / \mathrm{mm}^{2}$.

rées par des joints de flexion sur les plans $\{110\}$ et par des joints de torsion sur les plans $\{100\}$. - La taille de la sous-structure varie de façon inversement proportionnelle à la contrainte appliquée.

\subsection{ETUDE SEMI-QUANTITATIVE DE LA DÉSORIENTA-} TION DES SOUS-GRAINS. - Maurice et al. [2] ont proposé une méthode qui permet d'avoir une idée assez exacte du sens de la désorientation et de son ordre de grandeur à condition que les désorientations soient inférieures à $5^{\circ}$. Ce qui a été vérifié par diagramme de Laue.

Rappelons que le principe consiste à observer la variation de contraste d'une cellule lorsque le faisceau d'électrons la balaye sous différentes incidences le long d'une direction [1110] par exemple et à identifier cette variation dans un tableau d'étalonnage des variations de contraste en fonction de la désorientation.

Les résultats obtenus montrent que l'évolution

du contraste observé est compatible avec des blocs faiblement désorientés alternativement dans un sens et dans l'autre.

Un aspect qualitatif de la désorientation est mis en évidence (Fig. 5) sur une image obtenue à l'IRSID par M. Tixier montrant la déformation du diagramme de micro Kikuchi lorsque l'on surfocalise la sonde.

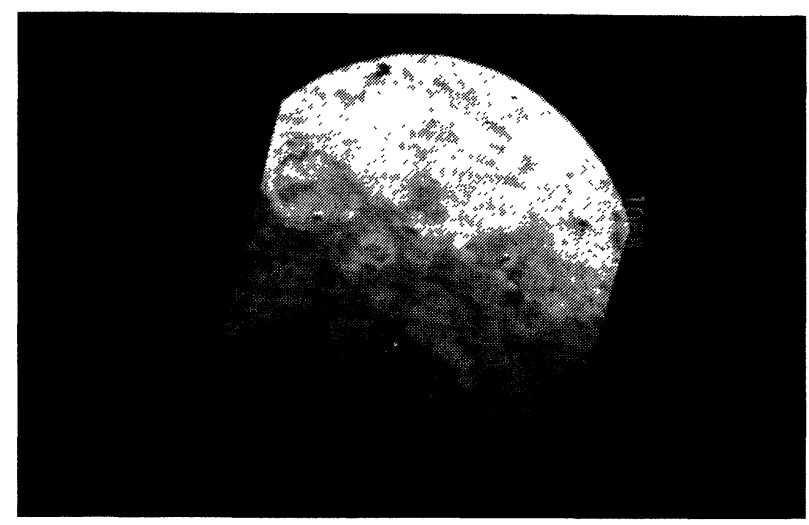

FIG. 5. - Microdiagramme de pseudo Kikuchi, sonde surfocalisée.

4. Conclusion. - Pour appliquer cette méthode avec plus de confiance il est nécessaire de quantifier les données utilisées, c'est-à-dire calculer les profils d'intensité pour les bandes de bas indice des cristaux étudiés, mesurer les variations du courant échantillon, réaliser quelques diagrammes de Kossel pour tester la courbe de variation de l'intensité en fonction de la désorientation.

Ce travail en cours actuellement permettra d'obtenir en peu de temps des informations quantitatives "sur la désorientation à partir desquelles on pourra tirer de précieux renseignements sur l'évolution des sousstructures et les mécanismes de polygonisation.

\section{Bibliographie}

[1] Dorrizi, P., Thèse Université Paris-Sud, Orsay (1973).

[2] Maurice, F., Zemskoff, A., Dorizzi, P., Poirier, J.-P., Micron 4 (1973) 184-192.

[3] Pitival, M., Blanchin, G., Vicario, E., Fontaine, G., (1971) Crystalline contrast and SEM channeling Patterns Proc., 25th Anniv. meeting of EMAG Inst. of Physics, 338, Cambridge. 